DOI: https://doi.org/10.11144/Javeriana.upsy18-4.rnof

\title{
Relación del nivel ocupacional, funcionamiento cognitivo y satisfacción vital en mayores de 55 años*
}

\section{Relationship of occupational level cognitive performance and life satisfaction in over 55 years of age}

Recepción: 10 Julio 2018 | Aceptación: 02 Noviembre 2019

\author{
José Manuel Aguilar-Parra \\ Universidad de Almería, España \\ ORCID: http://orcid.org/0000-0002-6703-0680 \\ RubÉN TRIGUEROS-RAmOS ${ }^{\mathrm{a}}$ \\ Universidad de Almería, España \\ ORCID: http://orcid.org/0000-0001-8489-5179 \\ David PAdilla-Góngora \\ Universidad de Almería, España \\ ORCID: http://orcid.org/0000-0002-5630-7074 \\ Remedios López-LiRia \\ Universidad de Almería, España \\ ORCID: http://orcid.org/0000-0002-3416-2791
} a Autor de correspondencia. Correo electrónico:
rtr088@ual.es

Para citar este artículo: Aguilar-Parra, J. M., Trigueros-Ramos, R., Padilla-Góngora, D., LópezLiria, R. (2019). Relación del nivel ocupacional, funcionamiento cognitivo y satisfacción vital en mayores de 55 años. Universitas Psychologica, 18(4), 1-9. https://doi.org/10.11144/Javeriana.upsy18-4.rnof

\section{RESUMEN}

El progresivo envejecimiento de la población obliga a las administraciones con competencias en materia sanitaria a intervenir en los efectos adversos de la falta de actividad física o funcional (depresión, ansiedad, deterioro cognitivo e insatisfacción vital, entre otros).

El objetivo de este estudio es comprobar si el nivel de ocupación en actividades básicas de la vida cotidiana u ocio de una muestra de personas mayores de 55 años tiene influencia sobre el grado de funcionamiento cognitivo y su satisfacción vital. Se trata de un estudio transversal, con un muestreo no probabilístico por conveniencia, desarrollado con 302 personas mayores de 55 años de varios centros participación activa (CPA) de la provincia de Almería (España). Los instrumentos utilizados para la valoración comprendieron el Mini-Exámen Cognoscitivo, la Escala de Satisfacción de Filadelfia y el Cuestionario de Conducta Ocupacional de Mayores (C-COM). El análisis multigrupo de los datos también incluyó un modelo estructural mediante path analysis. Los resultados han verificado la influencia de la ocupación en el deterioro cognitivo y la satisfacción vital, tanto en hombres como en mujeres. Las actividades de ocio aportan una mayor satisfacción vital que las ocupaciones del quehacer diario. La actitud hacia el envejecimiento ha sido el factor que ha arrojado una mayor correlación con el nivel ocupacional de la persona. Los datos respaldan la necesidad de unas recomendaciones adecuadas para el diseño de programas específicos que favorezcan intervenciones individuales y colectivas enfocadas al ejercicio, la prevención del deterioro cognitivo y el fomento de la satisfacción vital.

Palabras clave

ocupación; funcionamiento cognitivo; satisfacción vital; personas mayores; actividades cotidianas y de ocio. 


\begin{abstract}
The progressive aging of the population forces the administrations with competences in sanitary matters to intervene on the adverse effects of the lack of physical or functional activity (depression, anxiety, cognitive deterioration and vital dissatisfaction among others). The aim of this study was to verify if the level of occupation in basic activities of daily life or leisure of a sample of people over 55 years has influence on the degree of cognitive functioning and their life satisfaction. This is a cross-sectional study, with a non-probabilistic convenience sample carried out with 302 persons over 55 years of age from several active participation centres (CPA) in the province of Almería (Spain). The instruments used for the assessment included the Mini Cognitive Examination, the Philadelphia Satisfaction Scale and the Senior Occupational Behavior Questionnaire (C-COM). The multigroup analysis of the data also included a structural model using path analysis. The results have verified the influence of occupation on cognitive deterioration and life satisfaction in both men and women. Leisure activities provide greater life satisfaction than the occupations of daily life. The attitude toward aging has been the factor that has shown a greater correlation with the occupational level of the person. The data support the need for adequate recommendations for the design of specific programs that favor individual and collective interventions focused on exercise, the prevention of cognitive deterioration and the promotion of life satisfaction.

Keywords

occupation; cognitive functioning; life satisfaction; elderly; everyday and leisure activities.
\end{abstract}

\section{Introducción}

El progresivo envejecimiento de la población mundial y los destacados avances científicos justifican la importancia de que las personas mayores desarrollen un proceso activo caracterizado por altos niveles de ocupación, tanto en actividades de ocio, como en las de la vida diaria (AVD) (Liotta et al., 2018; Narushima, Liu, \& Diestelkamp, 2018; Sarabia, 2009). Existe un interés creciente por analizar y estudiar las principales políticas y programas que permitan promocionar y fomentar en estas personas actitudes positivas, así como implicarles activamente en un amplio abanico de actividades que los preparen para mantener el mayor nivel de ocupación posible, y les garantice una elevada satisfacción vital y un adecuado funcionamiento mental (Howe, 2009; Rotenberg \& Maeir, 2018; Tam, 2011). Todo ello contribuirá al desarrollo de un proceso de envejecimiento sólido y alejado de altos grados de deterioro cognitivo y de patologías de índole clínica, así como al fortalecimiento de los niveles de equilibrio emocional y de calidad de vida.

La formulación de políticas para lograr lo anterior continúa en auge, y trata de adaptarse a la sociedad e incluir acciones concretas como la seguridad económica, la actividad física, mental y social, y la participación laboral. En diversas comunidades autónomas de nuestro país la promoción de la capacidad funcional se realiza a través de ocio activo (el $83 \%$ a través de baile, teatro, música y pintura), fomento de la lectura, mantenimiento y rehabilitación cognitiva (talleres de memoria, cálculo) (Herrera, Muñoz, Martin, \& Cid, 2011). Según diversos autores (Fernández, Contreras, García, \& Villora, 2010; Gálvez, Caracuel, \& Jaenes, 2011; Kuska, 2018), los escasos índices de funcionalidad o actividad física plantean grandes limitaciones a la hora de ejecutar las actividades de la vida cotidiana y de ocio, una mayor dificultad para la correcta conservación de las funciones cognitivas, y el inadecuado equilibrio de los niveles de autoconcepto e imagen personal.

Estudios en población general (Gallego et al., 2012; Jessen-Winge, Petersen, \& Morville, 2018; Sun, Liao, Yang, \& Zeng, 2019) han logrado demostrar que el mantenimiento de un nivel de ocupación adecuado durante la vejez contribuye de manera decisiva a la mejora del estado general de salud y la calidad de vida del individuo, en consonancia con la evidencia actual que desea potenciar el envejecimiento activo a través de hábitos saludables y ejercicio físico, promoviendo los factores de protección del funcionamiento cognitivo, las emociones y un afrontamiento positivo, junto a la participación psicosocial. En España, cada comunidad desarrolla distintos talleres para la prevención de la dependencia (44.4\%), voluntariado social (44.4\%), ajedrez (16.7 \%) yoga, tai chi y psicoestimulación. Tres cuartas partes de los programas fomentan la formación y el desarrollo personal en colaboración con las universidades, actividades intergeneracionales y el acercamiento de los 
mayores a las tecnologías de la información y la comunicación (Herrera et al., 2011).

La práctica regular de ejercicio incrementa el nivel de actividad de las estructuras cognoscitivas, de la autoeficacia, la autoestima y el bienestar psicológico; además del funcionamiento fisiológico, el sistema cardiovascular, respiratorio e inmunológico, del aparato locomotor y de oxigenación de los tejidos, aspectos que van a contribuir en la movilidad, autonomía e independencia de los mayores a más largo plazo (Delle Fave et al., 2018; Living \& Tzonichaki, 2013; Moral, Orgaz, López, Amatria, \& Maneiro, 2018; RotenbergShpigelman \& Maeir, 2011). El $66.7 \%$ de los programas desarrollados en nuestro país promueven la autonomía a través de actividades físicas y deportivas, el $33.3 \%$ fomentan el acompañamiento, el $22.2 \%$ son programas de fisioterapia desarrollados principalmente en los centros de día, y el $16.7 \%$ corresponde a psicomotricidad (Herrera et al., 2011).

Partiendo de las evidencias empíricas descritas, se ha desarrollado esta investigación para analizar si el nivel ocupacional en actividades de la vida diaria u ocio de la persona mayor de 55 años tiene influencia sobre su funcionamiento cognitivo y nivel de satisfacción vital.

\section{Método}

\section{Participantes}

Se trata de un estudio transversal en una muestra compuesta por 302 personas mayores (129 hombres y 173 mujeres) con edades comprendidas entre los 55 y 92 años $(M=$ 69.73; DT $=9.63$ ), elegidos a través de un muestreo no probabilístico por conveniencia de entre la población asistente a Centros de días y Participación Activa de la provincia de Almería. Fueron encuestadas las personas que se encontraban en dichos centros realizando actividades de ocio y aceptaron participar voluntariamente en el estudio tras explicarles el objetivo del mismo.
El criterio de inclusión fue ser mayor de 55 años y aceptar participar voluntariamente, al firmar el consentimiento informado. Los criterios de exclusión fueron la no colaboración o ser incapaz de responder a las preguntas. Se contactó a los seis centros seleccionados para informar a los directores de los objetivos de la investigación y pedirles su colaboración. Se les solicitó el consentimiento informado y se administraron los cuestionarios bajo la supervisión de un encuestador experto que explicó la forma de cumplimentarlos, solucionando las dudas que surgieron durante el proceso. En todo momento se han respetado los principios éticos de la investigación durante la administración y el uso de la información recogida.

\section{Instrumentos}

Para valorar el deterioro cognitivo se utilizó el Mini-Examen Cognoscitivo (MEC)de Folstein, Folstein, y McHugh (1975), instrumento de cribaje para la detección de demencia o delirium, que cuantifica básicamente las funciones intelectuales (13 ítems que evalúan la orientación, memoria de fijación, memoria reciente, capacidad de concentración y de atención, identificación de objetos, órdenes verbales y escritas, abstracción, escritura y construcción). La puntuación total puede ir de 0 a 35. El estudio de fiabilidad ha demostrado una adecuada validez concurrente buena correlación, tanto en pacientes médicos $(r=0.86)$ como en pacientes psiquiátricos $(r=0.87)$, así como en la validez concurrente con el WAIS (Wechsler Adult Intelligence Scale), Matrices Progresivas de Raven y otros. En la Tabla 1 se ha reportado la fiabilidad de las variables en el estudio, calculada a través del Alpha de Cronbach.

\section{Procedimiento}

Para obtener el nivel ocupacional de la persona, se empleó el Cuestionario de Conducta Ocupacional de los Mayores (C-COM) de Aguilar-Parra (2012), que está compuesto por 7 ítems que extraen la conducta ocupacional de 
los mayores, tanto en su quehacer diario como en su tiempo de ocio: cómo viven, cuál es su nivel de salud percibida y de autonomía, número de visitas al médico, medicación que toman; y, finalmente, actividades que desempeñan. Es un cuestionario que se construyó ad hoc, validado interjueces, que obtuvo una buena fiabilidad y consistencia de las puntuaciones mediante el coeficiente de Cronbach y W de Kendall. Asimismo, fue validado en estudio piloto con 183 sujetos (Aguilar-Parra, 2012). En este artículo se han se han analizado los dos ítems de respuesta dicotómica (sí/no) que interrogan a la persona sobre el número de ocupaciones, tanto en AVD (rango de 1 a 10 ocupaciones) como en ocupaciones de ocio (rango de 1 a 26 ocupaciones); y la sumatoria de ambas puntuaciones, denominada Ocupación Total de la persona.

Se evaluó el bienestar subjetivo a través de la Escala de Satisfacción de Filadelfia, Philadelphia Geriatric Center Morale Scale (PGC) (Lawton, 1972), adaptada al castellano, que describe el estado de ánimo con 16 ítems donde se clasifica la moral en alta (puntuación $=1$ ), media (puntuación $=2$ ) y baja (puntuación $=3$ ).

\section{Análisis de datos}

Tras el análisis estadístico descriptivo de los datos, se testeó el modelo mediante path analysis (método de máxima verosimilitud junto con el procedimiento de bootstrapping) para evaluar las relaciones hipotéticas entre las variables del estudio.

Con el objetivo de aceptar o rechazar el modelo testeado, se utilizó un conjunto de varios índices de ajuste: $\chi 2 / g l, \quad$ CFI (ComparativeFitIndex), TLI (TuckerLewisIndex), IFI (IncrementalFitIndex), RMSEA

(RootMeanSquareErrorofApproximation) más su intervalo de confianza al $90 \%$, y SRMR (StandardizedRootMeanSquareResidual). Dado que el $\chi^{2}$ es muy sensible al tamaño muestral, se empleó el $\chi 2 / g l$, que considera aceptables valores inferiores a 5. Los índices incrementales (CFI,
TLI e IFI) muestran un buen ajuste con valores de 0.9 o superiores, mientras que los índices de error (RMSEA y SRMR) se consideran aceptables con valores iguales o menores de $0.08(\mathrm{Hu} \&$ Bentler, 1999). Para estos análisis se utilizaron los paquetes estadísticos SPSS 21.0 y AMOS 21.0.

\section{Resultados}

Relación entre nivel de ocupación de la persona, deterioro cognitivo y satisfacción vital

En la Tabla 1 se puede apreciar que el nivel de ocupación de los mayores de la muestra es bajo (se ha obtenido una media de 6 actividades de las 26 opciones posibles), y es ligeramente más alto en ocupaciones de actividades de la vida diaria (la media es algo superior a 4, de las 10 registradas). En relación con deterioro cognitivo, la media se encuentra en una puntuación que señala un estado cognitivo normal o ligero déficit. Por otro lado, se observa que las personas evaluadas mostraron una satisfacción vital media-baja en todos los factores, incluido el factor total (que se refiere a la agitación, la actitud hacia el propio envejecimiento y la insatisfacción con la soledad). Esta puntuación ha sido algo mayor cuando se analiza el factor insatisfacción con la soledad.

\section{Tabla 1}

Estadísticos descriptivos, fiabilidad y correlaciones entre las diferentes variables del estudio

\begin{tabular}{|c|c|c|c|c|c|c|c|c|c|c|c|c|c|}
\hline & Alpha & M & $D T$ & Asimetria & Curtosis & 1 & 2 & 3 & 4 & 5 & 6 & 7 & 8 \\
\hline $\begin{array}{c}\text { 1. Ocupación Total } \\
0.36\end{array}$ & 0.86 & 10.96 & 7.01 & 0.91 & -0.33 & - & $\begin{array}{l}0.906 \\
* * *\end{array}$ & $\begin{array}{l}0.982 \\
* * z \\
* * z\end{array}$ & 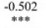 & $\begin{array}{l}-0.626 \\
* * * * \\
* 2\end{array}$ & $\begin{array}{l}-0.44 \\
* * *\end{array}$ & $\begin{array}{l}-0.726 \\
* * * \\
* 2\end{array}$ & 年 -0.024 \\
\hline 2. Ocupación AVDa & 0.83 & 4.44 & 2.25 & 0.52 & -0.62 & - & - & 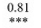 & $\begin{array}{l}-0.507 \\
*+\infty\end{array}$ & $\begin{array}{l}-0.535 \\
* *+*\end{array}$ & 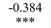 & 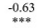 & $\begin{array}{l}-0.42 \\
*+*+\infty\end{array}$ \\
\hline 3. Ocupación Ocio & 0.78 & 6.52 & 5.06 & 0.98 & -0.19 & - & - & - & $\begin{array}{l}-0.47 \\
-0 * * *\end{array}$ & 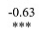 & $\begin{array}{l}-0.438 \\
* * * * \\
* 30\end{array}$ & 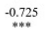 & 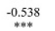 \\
\hline $\begin{array}{c}\text { 4. Deterioro Cognitivo } \\
1-5\end{array}$ & 0.81 & 1.72 & 0.84 & 1.07 & 0.67 & - & - & . & . & 0.394 & $\begin{array}{l}0.287 \\
* * * *\end{array}$ & $\begin{array}{c}0.457 \\
* * z=8\end{array}$ & $\begin{array}{l}0.391 \\
* * * * 1\end{array}$ \\
\hline 5. SV: Total. & 0.77 & 2.28 & 0.88 & -0.59 & -1.45 & - & - & - & - & - & 0.756 & 0.814 & 0.689 \\
\hline 6. SVb: Ag̣tación. & 0.75 & 2.24 & 0.8 & -0.45 & -1.29 & - & - & - & - & - & - & 0.527 & 0.487 \\
\hline $\begin{array}{l}\text { 7. Svb; Astitud hacia } \\
\text { envejeciminento. } \\
\text { 1-3. }\end{array}$ & -83 & 2.25 & 0.82 & -0.49 & -1.37 & - & - & - & - & - & - & - & $\begin{array}{c}0.638 \\
* * * *\end{array}$ \\
\hline $\begin{array}{l}\text { 8. SVb: Sataisfacción. } \\
1-3\end{array}$ & 0.8 & 1.76 & 0.82 & 0.47 & -1.38 & - & - & - & - & - & - & - & - \\
\hline
\end{tabular}

El análisis correlacional de Pearson muestra una asociación negativa moderada entre el nivel de ocupación de la persona y el deterioro cognitivo, lo que reflejaría que un mayor número de ocupaciones desarrolladas por la persona 
representa un menor deterioro cognitivo. Las tres medidas que se refieren al nivel de ocupación han mostrado una correlación negativa moderadacasi-alta, respecto al nivel de satisfacción vital de la persona. Teniendo en cuenta que las puntuaciones de este test de satisfacción vital funcionan de manera inversa, ya que 1 punto se corresponde con moral alta y 3 con baja, se interpreta que, a mayor ocupación, la satisfacción vital de la persona sería más alta. También se observa que las ocupaciones de ocio correlacionan de forma más intensa, y aportan una mayor satisfacción vital (Tabla 1).

Finalmente, se aprecia la existencia de correlación baja-casi-moderada, entre el funcionamiento cognitivo y la satisfacción vital de la persona.

El modelo hipotetizado (Figura 1) deseaba establecer si la sumatoria de ocupaciones desarrolladas por la persona predeciría de forma negativa y significativa tanto el nivel de satisfacción vital total como el grado de deterioro cognitivo. Los índices de ajuste obtenidos fueron satisfactorios: $\chi^{2}(1, N=302)=4.26, p=0.036$, $\chi^{2 / g l}=4.26, \mathrm{CFI}=0.95, \mathrm{IFI}=0.95, \mathrm{TLI}=0.94$, SRMR $=0.33$, RMSEA $=0.078$. Por lo tanto, los resultados del path analysis determinaron la relación existente entre la ocupación total y la prueba de deterioro cognitivo $(\beta=-0.5, p<$ $0.001)$ y entre ocupación total y satisfacción vital $(\beta=-0.62, p<0.001)$.

\section{Figura 1}

Modelo relacional del nivel ocupacional con el deterioro cognitivo y la satisfacción vital de la persona hipotetizado

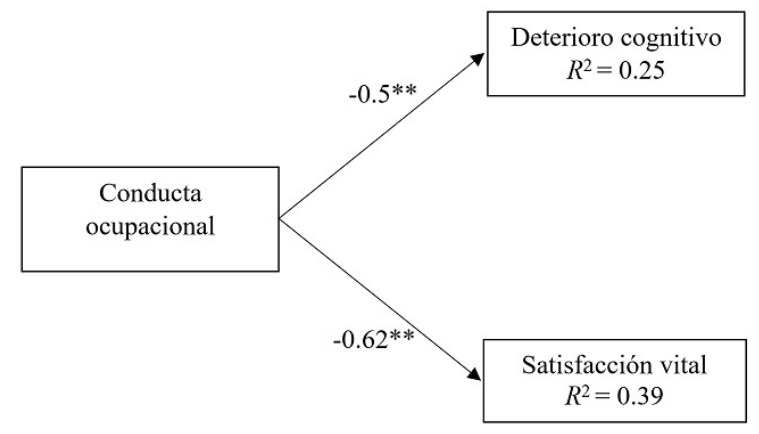

\section{Discusión}

El objetivo del presente estudio ha sido determinar si el nivel de ocupación de una muestra de mayores de 55 años influiría sobre su funcionamiento cognitivo y el nivel de satisfacción vital, cuestión que se ha verificado a través de una doble vía (correlación y path analysis).

Por un lado, se ha confirmado que existe una influencia moderada entre el nivel de ocupación de la persona y el funcionamiento cognitivo. En este sentido, los resultados estarían en consonancia con los obtenidos en otras investigaciones (Aguilar-Parra, Álvarez, Fernández-Campoy, Salguero, \& Pérez-Gallardo, 2013; Álvarez, Aguilar-Parra, \& MercaderRubio, 2012; Darwish, Farran, Assaad, \& Chaaya, 2018; Franco, Parra, González, Bernate, $\&$ Solis, 2013), que insisten en la idea de que aquellas personas que mantienen un cierto nivel de ocupación, tanto en actividades de la vida diaria como de ocio, van a experimentar un menor grado de deterioro cognitivo que aquellas otras personas que permanecen inactivas. Los factores de la autonomía funcional suelen estar, además, condicionados por las facultades cognitivas y emocionales y por las características del entorno, lo que influye en la capacidad del individuo para gestionar su propia vida. Previamente la literatura científica ha verificado que el ejercicio aeróbico regular y la formación permanente, son factores protectores ante el deterioro cognitivo y el riesgo de demencia.

Este estudio también señala que el nivel ocupacional influye sobre la satisfacción vital de las personas mayores, en consonancia con lo postulado por diversos autores que nos indican que elementos como la participación en actividades de ocio, la interacción social, y unas buenas redes sociales incrementan la función cognitiva y proporcionan apoyo emocional (Aguilar-Parra et al., 2013; Cancino, Rehbein-Felmer, \& Ortiz, 2018; Castellano, 2014; Meléndez, Navarro, Oliver, \& Tomás, 2009).

Aquí, además se describe que existe una mayor correlación entre los niveles de ocupación en 
José Manuel Aguilar-Parra, Rubén Trigueros-Ramos, David Padilla-Góngora, et al.

actividades de ocio y la satisfacción vital de los mayores, respecto a las actividades de la vida diaria. Diferencias que algunos investigadores han especulado que podrían deberse a que las actividades de ocio normalmente son elegidas por la persona de forma voluntaria para disfrutar, mientras que las actividades de la vida diaria no resultan tareas tan gratificantes (Allan, Wacholtz, \& Valdés, 2005; Castillo-Carniglia, Albala, Dangour, \& Uauy, 2012; FernándezPortero, Alarcón, \& Barrios-Padura, 2018). Por otro lado, este ocio implica un contacto frecuente con la gente que suele relacionarse con una vida activa, y con la sensación de protagonismo que protege ante la discapacidad. Esta relación también es a la inversa, ya que la diversidad de contactos sociales, la alta participación y productividad social predicen el mantenimiento de las actividades de la vida diaria.

En esta investigación la actitud hacia el envejecimiento ha sido el factor que ha arrojado una mayor correlación con el nivel ocupacional de la persona. Diferentes autores (FernándezGarcía \& Ponce, 2013) lo han vinculado al hecho de que la persona que realiza un mayor número de tareas se percibe a sí misma como más autónoma, más capacitada y, por tanto, no llega a interpretar el envejecimiento como algo negativo que pueda llegar a obstaculizar su devenir vital.

En relación con el deterioro o funcionamiento cognitivo, se obtienen correlaciones bajas, casi moderadas, con el nivel de satisfacción vital de la persona, como ya lo indican estudios previos (Blanco, Jiménez-Martí, PinazoHernándis, Sánchez-Sánchez, \& Torregosa-Ruíz, 2018; Rey, Canales, \& Táboas, 2011), que ratifican, junto con el modelo hipotetizado, que el nivel ocupacional de la personas se convierte en un predictor importante del funcionamiento cognitivo (Rabuñal et al., 2012; Noriega et al., 2019) y de la satisfacción vital (Ramírez $\&$ Lee, 2012). Finalmente, se ha confirmado, como en otros trabajos, que los efectos del nivel ocupacional sobre el funcionamiento cognitivo y el nivel de satisfacción vital se dan de forma similar, tanto en hombres como en mujeres (Cuadra-Peralta, Veloso-Besio, Puddu-Gallardo, Salgado-García, \& Peralta-Montecinos, 2012;
López \& Calero, 2009; Navarro, Calero, \& Calero-García, 2014).

Entre las limitaciones de este estudio se encuentra que la muestra únicamente representa a la población adulta mayor de la provincia de Almería. Esta se extrajo de diversos centros de día o participación activa de mayores, debido a que existe evidencia científica que respalda que la mayoría de las actividades se desarrollan en estos, espacios comunitarios que han demostrado ser un factor de éxito en los programas de promoción de la autonomía personal, la participación social y de promoción del envejecimiento saludable (Herrera et al., 2011). Por otro lado, la comparabilidad de resultados con relación a otras investigaciones publicadas sobre actividad en los mayores normalmente es compleja por la gran variedad de cuestionarios utilizados y la falta de consenso para definir las actividades ocupacionales o de ocio de las personas; por ello, para desarrollar este trabajo, se construyó y validó el cuestionario C-COM, con la finalidad de que fuera un instrumento sencillo y práctico para lograr los objetivos planteados. La utilización de este cuestionario construido ad hoc, simplemente para cuantificar el número actividades, tanto de ocio como de la vida diaria que desarrollan las personas, puede suponer también una limitación a tener en cuenta.

En España existen diferencias en la provisión de programas para la promoción de la autonomía personal, dependiendo del organismo competente (Imserso, ayuntamientos, entidades privadas concertadas, etc.). Como prospectiva para desarrollar futuras líneas de investigación, se evidencia la necesidad de desarrollar más estudios que generen consenso y establezcan un marco de iniciativas donde se definan estándares y criterios comunes para el desarrollo de programas efectivos que promuevan la actividad u ocupación de los mayores tras la jubilación (Herrera et al., 2011). Además, son precisos más estudios descriptivos, donde se analicen las necesidades de la población previa a la implantación de programas, para lo que es muy importante implicar a expertos, organizaciones $\mathrm{y}$ asociaciones de mayores. Es importante tener en cuenta aspectos socioculturales (sexo, 
cultura, situación familiar, experiencias vitales, formación) y la participación de estas personas mayores en la planificación, así como la ejecución y evaluación de dichos programas.

Este trabajo se ha desarrollado con la finalidad de dar a conocer cuál es la actividad habitual y de tiempo libre de una muestra de mayores de la provincia de Almería, datos que podrían facilitar unas recomendaciones adecuadas de promoción de la salud y el diseño de programas específicos que favorezcan intervenciones individuales y colectivas enfocadas a la prevención del deterioro cognitivo y el fomento de la satisfacción vital, acciones que contribuirían a mantener y mejorar las condiciones de vida que nos han llevado a ser uno de los países más longevos del mundo, y a retrasar la edad de inicio de la discapacidad.

Además, esta investigación respalda la idea de que existe la necesidad de crear departamentos de envejecimiento activo en cada ciudad, que organicen actividades que promuevan el ejercicio y una mayor participación social de las personas de más de 55 años, teniendo en cuenta sus preferencias, contexto, cultura, sus estilos de vida, etc., con el objeto de conseguir ciudadanos que conozcan y puedan controlar sus carencias físicas o funcionales y enfermedades, y transformarse en personas activas y saludables que disfruten de una vida autónoma y enriquecedora.

En definitiva, a la hora de desarrollar programas de promoción de la autonomía personal entre el colectivo de mayores, deberían priorizarse aquellas medidas que "acrediten" reforzar claramente el bienestar físico, mental, emocional y social, basadas en evidencia científica, y bajo los principios de equidad y accesibilidad, eliminando las barreras que dificultan la vida independiente.

\section{Referencias}

Aguilar-Parra, J. M. (2012). La conducta ocupacional, el proceso de toma de decisiones y su influencia en la calidad de vida de los mayores (Tesis Doctoral). Universidad de Almería, España.
Aguilar-Parra, J. M., Álvarez, J., FernándezCampoy, J. M., Salguero, D., \& PérezGallardo, E. R. (2013). Investigación sobre envejecimiento activo y ocupación como fuente de salud y calidad de vida. International Journal of Developmental and Educational Psychology: INFAD. Revista de Psicología, 2(1), 147-154.

Allan, N., Wachholtz, D., \& Valdés, A. (2005). Cambios en la Ocupación de los adultos mayores recientemente jubilados. Revista Chilena de Terapia Ocupacional, 5, 19-29.

Álvarez, J., Aguilar-Parra, J. M., \& MercaderRubio, I. (2012). Cognición y autonomía en personas mayores dependientes. Anales de Psicología, 28(3), 946-953.

Blanco, M., Jiménez-Martí, M., PinazoHernándis, S., Sánchez-Sánchez, F. J., \& Torregosa-Ruíz, M. (2018). Predictores de la satisfacción con la vida en personas adultas mayores en Costa Rica y España. Anales en Gerontología, 10(10), 1-35.

Cancino, M., Rehbein-Felmer, L., \& Ortiz, M. S. (2018). Funcionamiento cognitivo en adultos mayores: rol de la reserva cognitiva, apoyo social y depresión. Revista Médica de Chile, 146(3), 315-322. https://doi.org/10.4 067/s0034-98872018000300315

Castellano, C. L. (2014). La influencia del apoyo social en el estado emocional y las actitudes hacia la vejez y el envejecimiento en una muestra de ancianos. International Journal of Psychology and Psychological Therapy, 14(3), 365-377.

Castillo-Carniglia, Á., Albala, C., Dangour, A. D., \& Uauy, R. (2012). Factores asociados a satisfacción vital en una cohorte de adultos mayores de Santiago, Chile. Gaceta Sanitaria, 26(5), 414-420.

Cuadra-Peralta, A., Veloso-Besio, C., PudduGallardo, G., Salgado-García, P., \& PeraltaMontecinos, J. (2012). Effects of a positive psychology program in depressive symptoms and life satisfaction in the elderly. Psicologia: Reflexão e Crítica, 25(4), 644-652. https://doi.org/10.1590/S0102-79 722012000400003 
José Manuel Aguilar-Parra, Rubén Trigueros-Ramos, David Padilla-Góngora, et al.

Darwish, H., Farran, N., Assaad, S., \& Chaaya, M. (2018). Cognitive Reserve Factors in a Developing Country: Education and Occupational Attainment Lower the Risk of Dementia in a Sample of Lebanese Older Adults. Front. Aging Neuroscience, 10, 277. https://doi.org/10.3389/fnagi.2018.00277

Delle Fave, A., Bassi, M., Boccaletti, E. S., Roncaglione, C., Bernardelli, G., \& Mari, D. (2018) Promoting Well-Being in Old Age: The Psychological Benefits of Two Training Programs of Adapted Physical Activity. Frontiers in Psychology, 9, 828. http s://doi.org/10.3389/fpsyg.2018.00828

Fernández, J. G., Contreras, R. O., García, L. M., \& Villora, S. G. (2010). Autoconcepto físico según la actividad físicodeportiva realizada y la motivación hacia esta. Revista Latinoamericana de Psicología, 42(2), 251-263.

Fernández-García, T., \& Ponce, L. (2013). Envejecimiento activo: recomendaciones para la intervención social desde el modelo de gestión de casos. Portularia, 1, 87-97.

Fernández-Portero, C., Alarcón, D., \& BarriosPadura, A. (2018) Psychosocial indicators of habitability conditions and satisfaction with life among older people. Psyecology, 9(2), 152-176. https://doi.org/10.1080/217 11976.2017.1399573

Folstein, M. F., Folstein, S. E., \& McHugh, P. R. (1975). "Mini-mental state": a practical method for grading the cognitive state of patients for the clinician. Journal of Psychiatric Research, 12(3), 189-198.

Franco, M., Parra, E., González, F., Bernate, M., $\&$ Solis, A. (2013). Influencia del ejercicio físico en la prevención del deterioro cognitivo en las personas mayores: revisión sistemática. Revista de Neurología, 56(11), 545-554.

Gallego, J., Aguilar-Parra, J. M., Cangas, A. J., Torrecillas, L., Javier, J., Justo, C. F., \& Mañas, I. M. (2012). Programa de natación adaptada para personas mayores dependientes: beneficios psicológicos, físicos y fisiológicos. Revista de Psicología del Deporte, 21(1), 125-133.
Gálvez, J. G., Caracuel, J. C. C., \& Jaenes, J. C. J. (2011). Práctica de actividad física y velocidad de procesamiento cognitivo en mayores. Revista Internacional de Medicina y Ciencias de la Actividad Física y del Deporte, 11(44), 808-816.

Herrera, E., Muñoz, I., Martin, V., \& Cid, M. (2011). Spanish experiences to promote personal autonomy in the elderly. Gaceta Sanitaria, 25(2), 147-157.

Howe, D. (2009). A brief introduction to social work theory. Londres: Palgrave Macmillan.

Hu, L. T., \& Bentler, P. M. (1999). Cutoff criteria for fit indexes in covariance structure analysis: Conventional criteria versus new alternatives. Structural Equation Modeling: a Multidisciplinary Journal, 6(1), 1-55.

Jessen-Winge, C., Petersen, M. N., \& Morville, A. L. (2018). The influence of occupation on wellbeing, as experienced by the elderly: A systematic review. Database of Systematic Reviews $\mathcal{B}$ Implementation Reports, 16(5), 1174-1189. https://doi.org/10.11124/JBISR IR-2016-003123

Kuska, M. (2018). The Importance of Physical Activity in the Process of Successful Ageing - An Overview. Central. European Journal of Sport Sciences and Medicine, 21 (1), 55-65.

Lawton, M. P. (1972). The dimensions of morale. En D. P. Kent, R. Kastenbaum \& S. Sherwood (Eds.), Research, planning and action for the elderly (pp. 144-165). Nueva York, NY: Behavioral Publications.

Liotta, G., Canhao, H., Cenko, F., Cutini, R., Vellone, E., Illario, M., ...Marazzi, M. C. (2018). Active Ageing in Europe: Adding Healthy Life to Years. Frontiers in Medicine, 5, 123. https://doi.org/10.3389/fmed.2018. 00123

Living, D., \& Tzonichaki, I. (2013). Occupational therapy intervention in the ergonomic arrangement of the elderly's physical environment in order to maintain the safety in their. Epitheorese Klinikes Farmakologiaskai, 31(3), 227-233.

López, Á. G., \& Calero, M. D. (2009). Predictores del deterioro cognitivo en ancianos. Revista 
Española de Geriatría y Gerontología, 44(4), 220-224.

Meléndez, J. C., Navarro, E., Oliver, A., \& Tomás, J. M. (2009). La satisfacción vital en los mayores. Factores sociodemográficos. Boletín de Psicología, 95 (1), 29-42.

Moral, J. E., Orgaz, D., López, S., Amatria, M., \& Maneiro, R. (2018). Influencia de la actividad física en la autoestima y riesgo de dependencia en personas mayores activas y sedentarias. Anales de Psicología, 34(1), 162-166.

Narushima, M., Liu, J. Y., \& Diestelkamp, N. (2018). Lifelong learning in active ageing discourse: its conserving effect on wellbeing, health and vulnerability. Ageing ES Society, 38(4), 651-675.

Navarro, E., Calero, M. D., \& Calero-García, M. J. (2014). Diferencias entre hombres y mujeres mayores en funcionamiento cognitivo y calidad de vida. European Journal of Investigation in Health, Psychology and Education, 4(3), 267-277.

Noriega, C., Velasco, C., Pérez, G., Carretero, I., Chulián, A., \& López, J. (2019). Calidad de vida, bienestar psicológico y valores en personas mayores. Revista Clínica Contemporánea, 8(1), 1-13.

Rabuñal, R., Monte, R., Casariego, E., Pita, S., Pertega, S., \& Bal, M. (2012). Valoración de actividades básicas de la vida diaria y de deterioro cognitivo en centenarios: concordancia entre las escalas utilizadas. Revista de Calidad Asistencial, 27(1), 44-49.

Ramírez, M., \& Lee, S. L. (2012). Factores asociados a la satisfacción vital en adultos mayores de 60 años. Polis. Revista Latinoamericana, 11(33), 14-28. https://doi. org/10.4067/S0718-65682012000300020

Rey, A., Canales, I., \& Táboas, M. I. (2011). Calidad de vida percibida por las personas mayores. Consecuencias de un programa de estimulación cognitiva a través de la motricidad "Memoria en movimiento". Revista Española de Geriatría y Gerontología, 46(2), 74-80.

Rotenberg, S., \& Maeir, A. (2018). Occupationbased metacognitive group intervention for older adults experiencing memory problems: Feasibility study. British Journal of Occupational Therapy, 81(10). https://doi.o $\mathrm{rg} / 10.1177 / 0308022618760787$

Rotenberg-Shpigelman, S., \& Maeir, A. (2011). Participation-centered treatment for elderly with mild cognitive deficits: A "Book Club" group case study. Physical $\mathbb{E}$ Occupational Therapy in Geriatrics, 29(3), 222-232.

Sarabia, C. M. (2009). Envejecimiento exitoso y calidad de vida: Su papel en las teorías del envejecimiento. Gerokomos, 20(4), 172-174.

Sun, K., Liao, L., Yang, F., \& Zeng, Y. (2019). Analysis on Quality of Life of Elderly in Xiamen and Its Influencing Factors. Advances in Social Science, Education and Humanities Research. Actas del 3rd International Conference on Culture, Education and Economic Development of Modern Society (ICCESE). Moscú: Atlantis Press. https://doi.org/10.2991/iccese-19.20 19.137

Tam, M. (2011). Active ageing, active learning: policy and provision in Hong Kong. Studies in Continuing Education, 33(3), 289-299.

\section{Notas}

* Artículo de investigación. 